\title{
Long-term outcome of implants placed with guided bone regeneration (GBR) using resorbable and non-resorbable membranes after 12-14 years
}

\author{
Jung, Ronald E ; Fenner, Nadine ; Hämmerle, Christoph H F ; Zitzmann, Nicola U
}

\begin{abstract}
AIM: The aim of the present prospective study was to evaluate the long-term outcome of implants placed simultaneously with guided bone regeneration (GBR) using resorbable and non-resorbable membranes. MATERIALS AND METHODS: The original study population consisted of 72 patients receiving a total of 265 implants. In all GBR-treated sites, demineralized bovine bone mineral (DBBM) was used in combination either with a collagen (CM) or an Expanded polytetrafluoroethylene (e-PTFE) membrane. A total of 112 implants was treated with CM, 41 implants were treated with e-PTFE membranes, and 112 served as a control group because implants were entirely surrounded by bone and did not need any GBR procedures. Clinical and radiographic analyses were performed after a period of 12-14 years. RESULTS: The median follow-up time was 12.5 years (range 12-14 years). A total of 58 patients participated in the present investigation, corresponding to $80.5 \%$ of the original study population. The cumulative implant survival rate at the follow-up examination was $93.2 \%$. For the control group the cumulative survival rate was 94.6\%, for the CM 91.9\%, and for the e-PTFE 92.6\%. Differences among the groups were not statistically significant. The radiographically determined marginal bone level (MBL) amounted to: CM $2.36 \mathrm{~mm}$ (SD), e-PTFE $2.4 \mathrm{~mm}(\mathrm{SD})$, control $2.53 \mathrm{~mm}$ (SD). There is no evidence $(\mathrm{P}<0.2)$ that the slope of bone level over time is different for the three treatment groups. CONCLUSION: It is concluded that implants placed simultaneously with GBR procedures using resorbable or non-resorbable membranes reveal a high survival rate ranging from $91.9 \%$ to $92.6 \%$, therefore it is considered to be a safe and predictable therapy.
\end{abstract}

DOI: https://doi.org/10.1111/j.1600-0501.2012.02522.x

Posted at the Zurich Open Repository and Archive, University of Zurich ZORA URL: https://doi.org/10.5167/uzh-64940

Journal Article

Originally published at:

Jung, Ronald E; Fenner, Nadine; Hämmerle, Christoph H F; Zitzmann, Nicola U (2013). Long-term outcome of implants placed with guided bone regeneration (GBR) using resorbable and non-resorbable membranes after 12-14 years. Clinical Oral Implants Research, 24(10):1065-1073.

DOI: https://doi.org/10.1111/j.1600-0501.2012.02522.x 


\section{Long-term outcome of implants placed with guided bone regeneration (GBR) using resorbable and non- resorbable membranes after 12 to 14 years}

Ronald E. Jung ${ }^{\mathrm{a}}$, Nadine Fenner ${ }^{\mathrm{a}}$, Christoph H. F. Hämmerle ${ }^{\mathrm{a}}$, Nicola Zitzmann ${ }^{\mathrm{b}}$

${ }^{a}$ Department of Fixed and Removable Prosthodontics and Dental Material Science, University of Zurich, Switzerland

${ }^{\mathrm{b}}$ Clinic for Periodontology, Endodontology and Cariology, University of Basel, Switzerland

Key words: bone regeneration, membranes, bone transplantation, human, graft material, bone substitute, dental implant

Running title: Long-term outcome of GBR after 12-14 years

Address for correspondence: $\quad$ PD Dr. Ronald E. Jung

Department of Fixed and Removable

Prosthodontics and

Dental Material Science

Dental School, University of Zurich

Plattenstrasse 11

$\mathrm{CH}-8028$ Zurich, Switzerland

Phone: +4144634 3251

Fax: +4144634 4305

e-mail: ronald.jung@zzm.uzh.ch 


\section{Abstract}

Aim:

The aim of the present prospective study was to evaluate the long-term outcome of implants placed simultaneously with guided bone regeneration (GBR) using resorbable and non-resorbable membranes.

\section{Materials and Methods:}

The original study population consisted of 72 patients receiving a total of 265 implants. In all GBR treated sites, demineralized bovine bone mineral (DBBM) was used in combination either with a collagen (CM) or an e-PTFE membrane. A total of 112 implants were treated with CM, 41 implants were treated with e-PTFE membranes, and 112 served as a control group because implants were entirely surrounded by bone and did not need any GBR procedures. Clinical and radiographic analyses were performed after a period of 12 to 14 years.

\section{Results:}

The median follow up time was 12.5 years (range 12 to 14 years). A total of 58 patients participated in the present investigation, corresponding to $80.5 \%$ of the original study population. The cumulative implant survival rate at the follow up examination was $93.2 \%$. For the control group the cumulative survival rate was $94.6 \%$, for the CM $91.9 \%$, and for the e-PTFE it was $92.6 \%$. Differences among the groups were not statistically significant.

The radiographically determined marginal bone level (MBL) amounted to:

CM $2.36 \mathrm{~mm}$ (SD), e-PTFE $2.4 \mathrm{~mm}$ (SD), control 2.53mm (SD). There is no evidence $(p<0.2)$ that the slope of bone level over time is different for the three treatment groups.

\section{Conclusion:}

It is concluded that implants placed simultaneously with GBR procedures using resorbable or non-resorbable membranes reveal a high survival rate ranging from $91.9-92.6 \%$, therefore it is considered to be a safe and predictable therapy. 


\section{Introduction}

For implant placement in sites with insufficient bone quantity, different techniques have been established to reconstruct deficient alveolar ridges and to facilitate dental implant placement (Hämmerle et al. 2002)

These techniques typically include autogenouse bone grafting (Simion et al. 1998), distraction osteogenesis (Penarrocha-Diago et al. 2006; Walker 2005; Oda et al. 2004; Chiapasco \& Gatti 2004), bone splitting (Enislidis et al. 2006) and guided bone regeneration (GBR) (Dahlin et al. 1988; Dahlin et al. 1989; Nyman et al. 1990; Dahlin et al. 1991; Nyman \& Lang 1994; Hämmerle et al. 1996).

Among these, GBR is the most frequently used technique for bone regeneration in conjunction with or prior to implant placement. The principal idea of GBR is the use of membranes to exclude epithelial cells with a high turn over and to allow the migration of the desired cells (particularly osteoblasts) in the established wound space (Hämmerle \& Jung 2000). The successful use of non-resorbable and resorbable membrane materials for GBR has been documented in the literature (Hämmerle et al. 2002)

Expanded polytetrafluoroethylene membranes (e-PTFE) as barrier were regarded as standard for GBR due to its early and successful application (Dahlin et al. 1991; Davarpanah et al. 1991). Despite the high predictability of bone regeneration using ePTFE barriers, the main disadvantages of this material are that the membrane must be removed in a second surgical intervention and that membrane exposure can cause bacterial contamination (Simion et al. 1998; Machtei 2001). Subsequently, the inflammatory reaction of the surrounding soft tissues may require early removal of the membrane. To avoid these problems the use of biodegradable materials has been investigated. 
In the past $10-15$ years resorbable membranes made of collagen have become the membrane of choice in many clinical situations. However, clinical long-term data using these types of membranes are scare in dental literature.

Hence, the aim of the present prospective study was to evaluate clinical and radiological outcomes of implants placed simultaneously with guided bone regeneration (GBR) using resorbable and non-resorbable membranes after a period of 12 to 14 years.

\section{Material and Methods}

\subsection{Study design and original population}

In the present study patients have been followed clinically and radiologically over a period of 12 to 14 years. All patients were treated by specialists between 1994 and 1996 at the Department of Fixed and Removable Prosthodontics and Dental Material Science at the University of Zurich, Switzerland. Patients were preoperatively informed about the treatment modalities and gave their informed consent.

The original study population consisted of 72 patients as described in a previous study (Zitzmann et al. 2001). A subgroup with 24 out of these 72 patients was part of a former split-mouth study comparing two membranes (Zitzmann et al. 1997). The median age of the patients at the time of surgery was 56 years (range 18 - 74 years). 54 patients were women and 18 men. All patients have been recalled every 12 months for the first 5 years after implant placement between 1996 and 2001. Since then, it was recommended that they consult private offices for maintenance care.

For the present follow-up investigation, all patients were invited by an information letter to attend an appointment for clinical and radiographic assessment. When 
patients did not answer three weeks thereafter, they were called by phone in order to set an appointment.

\subsection{Dental implants and regeneration material}

A total of 265 implants were placed in 72 patients: 256 Brånemark (Nobel Biocare, Göteborg, Sweden), 8 Biomed 3i (Biomet 3i, Palm Beach Gardens, Florida), one IMZ $\left(\right.$ Friatec $^{\circledR}$ AG, Mannheim, Germany). Different implant lengths and diameters were used, with implant lengths varying between $6 \mathrm{~mm}$ and $20 \mathrm{~mm}$, and diameters from $3.75 \mathrm{~mm}$ to $5 \mathrm{~mm}$.

All test sites were treated with demineralized bovine bone mineral (DBBM) (Bio-Oss ${ }^{\circledR}$, Geistlich AG, Wolhusen, Switzerland) as a membrane supporting material and covered with either collagen membranes (CM) (Bio-Gide ${ }^{\circledR}$, Geistlich AG, Wolhusen, Switzerland) or e-PTFE membranes (Gore-Tex ${ }^{\circledR}$, W.L. Gore / Implant Innovations, West Palm Beach, FL). A total of 112 implants were treated with CM, in 41 implants e-PTFE membranes were applied, and 112 implants served as a control group because implants were entirely surrounded by bone and did not need any GBR procedures.

In the subgroup with 24 patients that was part of a former split-mouth study comparing two membranes (Zitzmann et al. 1997) randomization was carried out using Bio-Gide ${ }^{\circledR}$ and Gore-Tex ${ }^{\circledR}$ cards in sealed envelopes. One envelope was selected by each patient, and the choice on the card was applied to the defect site with the first (lowest) tooth position number using the American system. The second defect site was treated with the other membrane type. 


\subsection{Treatment protocol / surgical procedure}

Clinical procedures

The surgical procedures were described in detail in two previous publications (Zitzmann et al. 1997; Zitzmann et al. 2001). In brief, the implant site was prepared according to standard protocols for the Brånemark System. The exposed portions of all implant sites were grafted with DBBM. Each site was covered with the selected membrane, which was trimmed and adapted to the shape of the individual defect so that the membrane overlapped the defect circularly by a minimum of $2 \mathrm{~mm}$. The sites of implantation were primarily closed for a submerged healing. If exposure of the ePTFE membrane occurred and signs of inflammation were present, removal of the membrane was performed. If possible, the exposed e-PTFE membrane was kept in situ for a minimum period of 6 weeks. Re-entry was performed after 4 months of healing in the mandible and 6 months in the maxilla.

\section{Preoperative and Postoperative Medication}

One hour prior to surgery, patients received antibiotic prophylaxis $(750 \mathrm{mg}$ amoxicillin). The postoperative protocol included antibiotic prophylaxis $(375 \mathrm{mg}$ amoxicillin three times a day) for 5 days, rinsing with a $0.2 \%$ chlorhexidine solution twice daily for 10 days and a non-steroidal anti-inflammatory medication according to individual requirements.

\subsection{Follow-up examination}

The last follow-up examination was performed at the Clinic for Fixed and Removable

Prosthodontics and Dental Material Science, University of Zurich, Switzerland between the end of 2007 and 2009. Prior to the clinical and radiographic examination, a thorough medical history was obtained from each patient. 


\subsubsection{Personal questionnaire}

A questionnaire was used to evaluate patient's overall satisfaction concerning the treatment, aesthetics and function of the implants and the implant restorations on a visual analog (rating) scale from 0 to 10 , where 10 indicated the highest level of satisfaction.

\subsubsection{Clinical parameters}

The following parameters were examined during the clinical examination:

1. implant survival

2. presence / absence of plaque at the implants

3. probing pocket depths (PPD) were performed at six sites (mesio-buccal, midbuccal, disto-buccal, disto-lingual, mid-lingual, mesio-lingual) of each implant and recorded to the nearest millimeter with the periodontal probe UNC-15 using a force of 0.2-0.3 N (examiner N.F. was calibrated in advance).

4. bleeding on probing at six sites around the implant (BOP) (Ainamo \& Bay 1975)

5. height of the keratinized mucosa (KM) was measured with the periodontal probe UNC-15 at the buccal aspect of the implant

6. marginal soft tissue level (MSTL) measured from the top of the abutment (abutment-crown junction) to the soft tissue margin. Positive values described the abutment margin located in a supramucosal position, while negative values represented a submucosal position of the abutment-crown junction.

\subsubsection{Radiographic analysis}

For the evaluation of the marginal bone level, intraoral radiographs were taken using the long-cone paralleling technique with the central beam directed to the alveolar 
crest (Hawe x-ray film holder, Kerrhawe SA, Bioggio, Switzerland). The images were digitalized with a scanner (Epson Perfection V750 Pro) in a resolution of $1200 \mathrm{dpi.}$

The marginal bone level $(\mathrm{MBL})$, i.e. the distance between the top of the implant shoulder and the first visible bone-to-implant contact, was measured at the mesial and distal aspect with a 10-15x magnification (Buser et al. 1991; Weber et al. 1992) using an image analysis program (Image J64). From the two measured values on the mesial and distal aspect, the greater distance was used for further analyses. The measured distance between three implant threads was used as the basis for the calibration and determination of the exact magnification and distortion of the images (Rodoni, et al. 2005). All measurements were performed by two examiners to the nearest $0.1 \mathrm{~mm}$. In case of disagreement the evaluation was redone and results discussed until an agreement was found.

\subsubsection{Data presentation and statistical analysis}

Statistics and plots were produced with R [Version R version 2.10.1 (2009-12-14), R (2005)]. By preference, 95\%-confidence intervals computed from mixed-model contrasts were use to qualify the results and the group differences.

Numerical estimates and confidence intervals in this paper were obtained from linear mixed models (Pinheiro and Bates, 2000) or from mixed-model Cox regression (Therneau 2009) to handle correlation due to multiple implants per subject. Assumptions for linear mixed models were checked by inspection of residual plots.

To obtain comparable estimates for the observed variables given the spread of follow-up times, a linear mixed growth model was fitted to the data (Pinheiro and Bates 2000). Parameter "treatment" with three levels "Control", "CM" and "e-PTFE" 
was used as a fixed categorical effect, while time in years was taken as continuous variable. To adjust for correlations with multiple implants in the same patient, the parameter "implant within patient" was used as a random factor. For follow-up times of 3 years and above a linear regression model was applied. Data from earlier visits were not used in the analysis. The validity of the linearity assumption was tested for all variables. The linear mixed model allows for adequate consideration of correlations in the study design, since some comparisons are within subject (splitmouth), and others are between subjects (randomized). The mixed-model effectively computes a linear regression line for each individual implant, and uses the predictions of these interpolations as a new statistical data set. It takes into account that data from one subject are more similar to each other than between subjects (i.e. the within-subject correlation), and that the within-subject differences have smaller variances compared to between-subject differences.

From the linear model, predictions for a follow-up time of 12 years and their betweentreatment pairwise differences were computed, and confidence intervals were given.

For the analysis of the implant survival (time to implant loss), the full (intention-totreat (ITT) data set was used; for dropouts, the last known implant survival time was censored.

The ITT subset consists of all data collected in the study published in 2001 (Zitzmann et al. 2001) and the present study, and includes patients who were early losses of dropouts. The full subset was used for Cox regression analysis of implant survival only, and applied the last date recorded for implant function as the possibly censored survival time. This ITT subset comprised 72 patients with 265 implants. 


\section{Results}

\subsection{Follow-up Outcomes}

\subsubsection{Study Population and Drop Out}

A total of 58 patients with 222 implants participated in the present study, which represents $80.5 \%$ of the original study population consisting of 72 patients. 45 patients were women and 13 men. The median age at the time of re-examination was 67.5 years (range 32-87 years). 14 patients could not be examined. 6 out of these 14 patients died and 8 patients could not be reached for re-examination because of geographic reasons or severe illness. One of the subjects (age 88) who was not re-examined because of illness confirmed by phone that the implant reconstruction was in place without any problems. All patients were examined by one examiner, except of 6 subjects who went to their private dentists and gave their approval to transfer clinical data and radiographs. One patient refused to take current radiographs at the examination but the dentist confirmed that all implants were stable and without clinical symptoms or radiologic diagnoses. Therefore, a total of 222 implants could be reassessed after a median observation period of 12.5 years (range 12 to 14 years).

Of the 43 implants in 14 patients that could not be evaluated, 21 were treated with CM, 3 with e-PTFE, and 19 were part of the control group.

The distribution of the implant location in the upper and the lower jaw was very homogeneous (Table 1). The majority of implants was restored with fixed dental prostheses. The proportions of the type of restoration were different in each of the three treatment groups and are shown in Table 2. 


\subsubsection{Health questionnaire}

Due to the increased age of the population (median age 67.5 years) a high incidence of different diseases was reported. These include three patients with diabetes mellitus, one patient with breast cancer, three patients with cerebral apoplexy, one with a heart attack and one with Alzheimer's disease. Patient's history revealed that seven individuals smoked and seven did not answer this question.

\subsubsection{Personal questionnaire}

The evaluation of patients' satisfaction with the implants and the restoration was 10 on a scale of 10 for $76 \%$ implants; for $9 \%$ of the implants, satisfaction was $0 / 10$.

\subsubsection{Clinical examinations}

\section{Implant survival}

The cumulative implant survival rate at the 12.5 years follow-up examination was $93.2 \%$ with 18 implants lost out of 265.

In the control group 6 implants out of 112 were lost and the cumulative survival rate was $94.6 \%$. In the CM group 9 implants out of 112 were lost with a survival rate of 91.9\%. In the e-PTFE group 3 implants out of 41 were lost, the survival rate was 92.6\% (Table 3 and Fig. 1). Statistically there is no evidence for differences in implant survival among the three treatment groups.

\section{Probing pocket depth (PPD)}

In the original study probing pocket depth was not measured. The present study revealed that the probing depth in the maxilla were $0.7 \mathrm{~mm}$ higher than in the mandible ( $p=0.0039$, Table 4). However, there is no evidence that probing depth was different among the three treatment groups (Table 5). 
The mean height of keratinized mucosa on the buccal aspect was higher in the maxilla than in the mandible. Estimated values of keratinization at 12.5 years followup are given in Table 6.

Marginal soft tissue level (MSTL)

$49 \%$ of all crown margins at the mid-buccal aspect were not visible after 12.5 years and were located either in a submucosal position $(26 \%)$ or at the abutment-crown junction (23\%) (Fig. 2-4). In contrast, $51 \%$ of the crown margins ware visible and were located in a supramucosal position (Fig. 5-7).

The estimated MSTL at the 12.5 years follow-up for the e-PTFE group was significantly closer to zero compared to the control group by $-0.32 \mathrm{~mm}(p=0.01)$, for the CM group it is marginally closer to zero compared to the control group by -0.17 $\mathrm{mm}(p=0.06)$. For all treatments the regression is estimated to progress at 0.068 $\mathrm{mm} /$ year, with a $95 \%$-confidence interval of 0.047 to $0.089 \mathrm{~mm} / \mathrm{year}$ (Table 7 ).

\subsubsection{Radiological examinations}

The radiographic evaluation demonstrated that all but two implants were radiographically integrated indicated by a direct contact between the bone and the implant. Two implants revealed an almost complete bone loss and were considered as implant failures (Fig. 8). For the statistical analysis these two implants were counted as implant losses and are included in Table 3 and Fig. 1.

The mean distance of the first bone-to-implant contact to the implant-abutment junction amounted to $2.36 \mathrm{~mm}$ for the CM group, $2.4 \mathrm{~mm}$ for the e-PTFE group and to $2.53 \mathrm{~mm}$ for the control group (Table 8 ). There was no evidence that the slope of the bone level over time was differed among the three treatment groups $(p<0.2)$ (Table 
9). The average change in bone level over time was $0.081 \mathrm{~mm} / \mathrm{year}$, with a $95 \%$ confidence interval of 0.072 to $0.09 \mathrm{~mm} / \mathrm{year}$ (Fig.9).

The correlation between the marginal bone level and the marginal soft tissue level is shown in Figure 10. With greater distances from the bone-to-implant contact to the implant-abutment junction, higher marginal soft tissue levels were found.

\section{Discussion}

The present prospective study evaluated the clinical and radiographic outcomes of implants placed simultaneously with guided bone regeneration (GBR) using resorbable and non-resorbable membranes after a period of 12.5 years. Similar survival rates for implants placed with or without bone augmentation were demonstrated, and results did not depend on the membrane material used. With 18 implant losses out of $265(7 \%)$, all treatment modalities revealed a generally low number of implant failures.

A lot of effort was given to recall as much patients as possible for the present longterm study even if they had not been in regular maintenance care for the last eight years. Therefore, $80.5 \%$ of the entire study population could be reassessed. This is comparable to a recent long-term study documenting a value of $84.2 \%$ of recalled patients after 17-19 years (Bergenblock, et al. 2010). Another clinical long-term investigation included $72 \%$ of the original study group after $10-16$ years (Simonis, et al. 2010).

One major interest in longitudinal studies is the survival rate. The present study showed an overall survival rate of $93 \%$ after 12.5 years. This is higher compared to a recent long-term study showing a survival rate of $89.3 \%$ after 10 years and a 
cumulative survival rate up to 16 years of $82.9 \%$ (Simonis et al. 2010). Another human study demonstrated a cumulative survival rate of $96.8 \%$ after an average follow-up time of 18 years (Bergenblock, et al. 2010). In contrast to the aforementioned studies, the present investigation included implants placed simultaneously with bone augmentation procedures using 2 different types of membranes. To our knowledge, there are presently no other long-term studies evaluating implants placed in regenerated bone using different types of membranes.

Two systematic reviews dealt with survival rates of implants placed in regenerated bone (Hammerle et al. 2002; Donos et al. 2008). In the more recent review, the implant survival rate at the augmented sites varied irrespective of the procedure from $91.7 \%$ to $100 \%$ and from $93.2 \%$ to $100 \%$ at the control sites for a period between 12 and 59.1 months (Donos et al. 2008). In the review from 2002, the survival rate of implants placed into sites with augmented bone using barrier membranes varied between $79 \%$ and $100 \%$ with the majority of studies indicating more than $90 \%$ survival after at least one year of function (Hammerle et al. 2002). In this systematic review three studies were included reporting on 5-year data of implants placed in regenerated bone (Buser et al. 1996; Becker et al. 1999; Zitzmann et al. 2001). Survival rates were $79.4 \%$ for implants with dehiscence/fenestration defects treated with e-PTFE membranes and $93.9 \%$ for implants in extraction sites treated with ePTFE membranes (Becker et al. 1999) and 100\% for implants treated with e-PTFE membranes (Buser et al. 1996). In the third study, the survival rate was $92.6 \%$ for implants treated with e-PTFE membranes and $95.4 \%$ for implants treated with collagen membrane (Zitzmann et al. 2001). The latter included the same patient population as the present study. Based on all these clinical data the present study revealed a quite high implant survival rate after a mean observation period of 12.5 years. 
Another important parameter of longitudinal studies is the radiographic change in marginal bone level over time. The present study revealed radiographic bone changes from 6 months until the follow-up of at least 12 years. It seems to be confirmed that marginal bone level changes take place particularly within the first 12 months after implant placement and functional loading. Thereafter, the bone level changes reached a plateau revealing minimal bone loss over time (Zitzmann et al. 2001. The radiographic examination in the present study showed that the average bone loss over the mean observation period of 12.5 years was $0.081 \mathrm{~mm} /$ year, with a $95 \%$-confidence interval of 0.072 to $0.09 \mathrm{~mm} /$ year. The estimated radiological bone level after 12.5 years resulted in $2.36 \mathrm{~mm}$ for the control group, $2.4 \mathrm{~mm}$ for the $\mathrm{CM}$ and $2.53 \mathrm{~mm}$ for the e-PTFE group. Keeping the average bone loss of $0.081 \mathrm{~mm} /$ year in mind, the marginal bone level in the present investigation seems to be comparable to studies with shorter observation periods. A recently published retrospective crosssectional study reported about marginal bone levels of $1.73 \mathrm{~mm}$ for the group without GBR procedures and $1.83 \mathrm{~mm}$ for the test group with bone augmentation after only 5 years (Benic et al. 2009). Another clinical study measured a mean marginal bone level at implants treated with GBR of $2.05 \mathrm{~mm}$ after 5 years (Blanco et al. 2005).

Limited data are available on studies comparing the clinical and radiographic outcome of implants placed with simultaneous GBR with implants placed in pristine bone. Two systematic reviews and one clinical study reported no difference in terms of radiographic marginal bone level between implants placed in pristine bone and implants combined with bone regeneration procedures (Hammerle et al. 2002; Donos et al. 2008, Benic et al. 2009). In the present study the sites treated with e-PTFE membrane showed slightly more bone loss compared to the other two groups. This might be explained by the fact that the sites treated with e-PTFE revealed more soft 
tissue complications and membrane exposures within the early healing phase (Zitzmann et al. 1997).

Several previous clinical studies reported a reduced amount of bone regeneration in sites demonstrating early membrane exposure and removal compared to sites with uneventful healing (Becker et al. 1999; Simion et al. 1994; Moses et al. 2005).

The marginal soft tissue level (MSTL) has been assessed after 6 months until the final examination at 12.5 years in the present study. It would be of clinical importance to predict bone loss by clinical measurements of the MSTL or the height of keratinized mucosa. The statistical analysis revealed a significant correlation between the marginal bone level and MSTL $\left(p=8.4 \times 10^{-5}\right)$. However, these results have to be interpreted with caution. Even if the predictive effect is present, the correlation between bone level and MSTL is low with $r=0.32$. In contrast, the statistics shows no evidence that the height of keratinized mucosa is a predictor for bone loss. In general, one of the intentions of performing GBR procedures at an implant site is to maintain the marginal soft tissue level at the buccal aspect. The present study revealed no difference between MSTL at the control vs. CM or ePTFE sites. Hence, it can be speculated that the augmented bone, irrespective of the preoperative defect morphology, was able to support the soft tissue in a similar way as the native bone. However, it is important to mention that $51 \%$ of the crown margins were visible and were located in a supramucosal position after a mean follow-up time of 12.5 years. The data did not demonstrate any difference between the groups revealing a similar behavior of sites with GBR and without GBR in terms of MSTLs. Statistically, there is no evidence that patient satisfaction and MSTL are correlated. 
It is concluded that implants placed simultaneously with GBR procedures using resorbable or non-resorbable membranes reveal a high survival rate ranging from 91.9-92.6\%. There was no statistical significant difference compared to implants placed in pristine bone without GBR procedures demonstrating an implant survival rate of $94.6 \%$ after 12.5 years of observation. Clinically and radiologically there was no difference between resorbable collagen membranes or non-resorbable e-PTFE membranes, considering these procedures as safe and predictable therapies over a long-term follow-up time of 12-14 years.

\section{Acknowledgement}

The authors express their special thanks to Dr. Dieter Menne (Menne Biomed Consulting, D-72074 Tübingen) for performing the statistical analysis and providing continuous expertise and support throughout the manuscript preparation. This study has been supported and founded by the Clinic for Fixed and Removable Prosthodontics, University of Zürich, Switzerland.

\section{References}

Ainamo, J. \& Bay, I. (1975) Problems and proposals for recording gingivitis and plaque. International dental journal 25: 229-235.

Becker, W., Dahlin, C., Lekholm, U., Bergstrom, C., van Steenberghe, D., Higuchi, K. \& Becker, B. E. (1999) Five-year evaluation of implants placed at extraction and with dehiscences and fenestration defects augmented with eptfe membranes: Results from a prospective multicenter study. Clinical implant dentistry and related reserach 1: $27-32$.

Benic, G. I., Jung, R. E., Siegenthaler, D. W. \& Hammerle, C. H. (2009) Clinical and radiographic comparison of implants in regenerated or native bone: 5-year results. Clinical Oral Implants Research 20: 507-513. 
Bergenblock, S., Andersson, B., Furst, B. \& Jemt, T. Long-term follow-up of ceraone single-implant restorations: An 18-year follow-up study based on a prospective patient cohort. Clinical implant dentistry and related reserach.

Blanco, J., Alonso, A. \& Sanz, M. (2005) Long-term results and survival rate of implants treated with guided bone regeneration: A 5-year case series prospective study. Clinical Oral Implants Research 16: 294-301.

Buser, D., Dula, K., Lang, N. P. \& Nyman, S. (1996) Long-term stability of osseointegrated implants in bone regenerated with the membrane technique. 5-year results of a prospective study with 12 implants. Clinical Oral Implants Research 7: 175-183.

Chiapasco, M. \& Gatti, C. (2004) Immediate loading of dental implants placed in revascularized fibula free flaps: A clinical report on 2 consecutive patients. The International journal of oral \& maxillofacial implants 19: 906-912.

Dahlin, C., Andersson, L. \& Linde, A. (1991) Bone augmentation at fenestrated implants by an osteopromotive membrane technique. A controlled clinical study. Clinical Oral Implants Research 2: 159-165.

Dahlin, C., Lekholm, U. \& Linde, A. (1991) Membrane-induced bone augmentation at titanium implants. A report on ten fixtures followed from 1 to 3 years after loading. The International journal of periodontics \& restorative dentistry11: 273-281.

Dahlin, C., Linde, A., Gottlow, J. \& Nyman, S. (1988) Healing of bone defects by guided tissue regeneration. Plastic and reconstructive surgery 81: 672-676.

Dahlin, C., Sennerby, L., Lekholm, U., Linde, A. \& Nyman, S. (1989) Generation of new bone around titanium implants using a membrane technique: An experimental study in rabbits. The International journal of oral \& maxillofacial implants 4: 19-25.

Davarpanah, M., Tecucianu, J. F., Slama, M. \& Celletti, R. (1991) [bone regeneration in implantology. The use of gore-tex membranes: Gtam]. Journal de Parodonologie 10: $169-176$.

Donos, N., Mardas, N. \& Chadha, V. (2008) Clinical outcomes of implants following lateral bone augmentation: Systematic assessment of available options (barrier membranes, bone grafts, split osteotomy). Journal of clinical periodontology 35: 173202.

Enislidis, G., Wittwer, G. \& Ewers, R. (2006) Preliminary report on a staged ridge splitting technique for implant placement in the mandible: A technical note. The International journal of oral \& maxillofacial implants 21: 445-449.

Hämmerle, C. H. F., Jung, R. E. Bone augmentation by means of barrier membranes. Periodontology 2000; 2003; 33: 36-53

Hammerle, C. H., Jung, R. E. \& Feloutzis, A. (2002) A systematic review of the survival of implants in bone sites augmented with barrier membranes (guided bone 
regeneration) in partially edentulous patients. Journal of clinical periodontology 29 Suppl 3: 226-231; discussion 232-223.

Hammerle, C. H., Schmid, J., Olah, A. J. \& Lang, N. P. (1996) A novel model system for the study of experimental guided bone formation in humans. Clinical Oral Implants Research 7: 38-47.

Machtei, E. E. (2001) The effect of membrane exposure on the outcome of regenerative procedures in humans: A meta-analysis. Journal of periodontology $\mathbf{7 2}$ : 512-516.

Moses, O., Pitaru, S., Artzi, Z. \& Nemcovsky, C. E. (2005) Healing of dehiscencetype defects in implants placed together with different barrier membranes: A comparative clinical study. Clinical Oral Implants Research 16: 210-219.

Nyman, S., Lang, N. P., Buser, D. \& Bragger, U. (1990) Bone regeneration adjacent to titanium dental implants using guided tissue regeneration: A report of two cases. The International journal of oral \& maxillofacial implants 5: 9-14.

Nyman, S. R. \& Lang, N. P. (1994) Guided tissue regeneration and dental implants. Periodontology 2000 4: 109-118.

Oda, T., Suzuki, H., Yokota, M. \& Ueda, M. (2004) Horizontal alveolar distraction of the narrow maxillary ridge for implant placement. Journal of oral and maxillofacial surgery 62: 1530-1534.

Penarrocha-Diago, M., Gomez-Adrian, M. D., Garcia-Garcia, A., Camacho-Alonso, F. \& Rambla-Ferrer, J. (2006) Vertical mandibular alveolar bone distraction and dental implant placement: A case report. The journal of oral implantology 32: 137-141.

Pinheiro, JC Bates DM (2000). Mixed-Effects Models in S and S-Plus. ISBN 0-38798957-0. Springer (cit. on pp. 6, 24).

Rodoni, L. R., Glauser, R., Feloutzis, A. \& Hammerle, C. H. (2005) Implants in the posterior maxilla: A comparative clinical and radiologic study. The International journal of oral \& maxillofacial implants 20: 231-237.

Simion, M., Baldoni, M., Rossi, P. \& Zaffe, D. (1994) A comparative study of the effectiveness of e-ptfe membranes with and without early exposure during the healing period. The International journal of periodontics \& restorative dentistry14: $166-180$

Simion, M., Jovanovic, S. A., Trisi, P., Scarano, A. \& Piattelli, A. (1998) Vertical ridge augmentation around dental implants using a membrane technique and autogenous bone or allografts in humans. The International journal of periodontics \& restorative dentistry 18: 8-23.

Simonis, P., Dufour, T. \& Tenenbaum, H. Long-term implant survival and success: A 10-16-year follow-up of non-submerged dental implants. Clinical Oral Implants Research 21: 772-777. 
Therneau, T. (2009). Cox proportional hazards models containing Gaussian random effects, also known as frailty models. Mayo Clinic, Rochester. url: http://r-forge.r-project.org.

Walker, D. A. (2005) Mandibular distraction osteogenesis for endosseous dental implants. J Canadian dental association71: 171-175.

Zitzmann, N. U., Naef, R. \& Scharer, P. (1997) Resorbable versus nonresorbable membranes in combination with bio-oss for guided bone regeneration. The International journal of oral \& maxillofacial implants 12: 844-852.

Zitzmann, N. U., Scharer, P. \& Marinello, C. P. (2001) Long-term results of implants treated with guided bone regeneration: A 5-year prospective study. The International journal of oral \& maxillofacial implants 16: 355-366. 